\title{
Impact of a Radio Access Network Capacity on the HSDPA Link Performance
}

\author{
Xinzhi Yan, Jamil Y. Khan \\ School of Electrical Engineering \& Computer Science \\ The University of Newcastle \\ Callaghan, NSW 2308, Australia \\ Xinzhi.Yan@studentmail.newcastle.edu.au, \\ Jamil.Khan@newcastle.edu.au
}

\author{
Brendan Jones \\ Mobile Network Quality \\ SingTel OPTUS \\ Sydney, NSW, Australia
}

\begin{abstract}
This paper analyzes the impact of the $I_{u b}$ link characteristics on the HSDPA air interface performance. In this work three different transport protocols are used to examine the effect of the $I_{u b}$ link on the HSDPA air interface. The paper uses both analytical and simulation techniques to analyze the relationship between the HSDPA air interface performance and the $I_{u b}$ link characteristics. The paper uses a Markov chain based analytical model and an OPNET based simulation model to obtain HSDPA air interface performance results.
\end{abstract}

Keywords HSDPA; Flow control; ATM; IP; 3GPP; UTRAN

\section{INTRODUCTION}

The UMTS radio network standard has continued to evolve under the 3GPP Long Term Evolution (LTE) plan. As one of the initial steps of the evolution the 3GPP has introduced the HSDPA (High Speed Downlink Packet Access) standard for downlink data and multimedia traffic [1]. The HSDPA architecture was initially introduced in the $3 \mathrm{GPP}$ release 5 . The HSDPA channel uses a short $2 \mathrm{~ms}$ transmission time interval (TTI), and uses variable coding and modulation techniques to achieve high data rate. In addition to above techniques the Node-B based fast scheduling and fast physical layer hybrid automatic repeat request (H-ARQ) techniques are used in the standard. According to the HSDPA specifications within next few years the air interface data rates could reach up to 14.4 Mbps. The peak data rate requires extremely favorable radio conditions and it is unlikely that all users in a cell would be able to get such a high data rate. In a HSDPA network user data rate will vary due to transmission conditions, and also as a result of time and code sharing among users. When the average traffic on the $I_{u b}$ link is less than the peak rate from the efficiency point of view it is not economical to allocate $I_{u b}$ link bandwidth at a peak rate. The overall HSDPA network throughput and QoS (Quality of Service) will depend on the air interface and radio link performances. For a wireless packet based multimedia services the radio access networks can significantly influence the performance of an air interface. The impact of the radio access network will increase with increasing air interface data rates.

The HSDPA architecture uses a downlink buffer in the Node-B. The buffer along with a scheduler that shares the transmission resources enables a higher peak transmission rate for HSDPA users on a radio link. A flow control is generally employed on the $\mathrm{I}_{\mathrm{ub}}$ link between a RNC (Radio Network Controller) and a Node-B to avoid buffer overflow or buffer starvation. If an $\mathrm{I}_{\mathrm{ub}}$ link cannot provide high enough instantaneous bandwidth, sufficient number of packets cannot be supplied to the Node-B from the RNC. Consequently, there may not be enough packets in Node-B buffer to sustain a peak transmission rate on the air interface [2]. Instantaneous bandwidth of a link could be affected by the transport protocol architecture and its physical transmission rate. The constraint of the $\mathrm{I}_{\mathrm{ub}}$ link bandwidth could degrade the performance of a HSDPA air interface. Hence, it is necessary to study the impact of the $\mathrm{I}_{\mathrm{ub}}$ link characteristics for the HSDPA air interface, which is the major objective of this paper.

The traffic flow between a RNC and a Node-B is controlled by the frame transmission protocol on the $\mathrm{I}_{\mathrm{ub}}$ link. Currently several transport protocols exist which can be deployed on the $\mathrm{I}_{\mathrm{ub}}$ link. In the release 99 of 3GPP, the AAL2/ATM transport technology was chosen to transmit multimedia traffic on the $\mathrm{I}_{\mathrm{ub}}$ interface. Later, in the 3GPP release 5, an IP-based transport protocol has been identified as the appropriate transport technology for the next generation network that can cater for multimedia traffic. In addition to above recently the Carrier Class Ethernet has attracted a lot of attention of network researchers for the wide area network deployment because of relatively lower cost, simplicity and flexibilities. Transport protocols of a radio access network can affect the performance of an air interface on the downlink. This issue is also investigated in this.

Reviewing the literature in this area, we found several works have concentrated on the $\mathrm{I}_{\mathrm{ub}}$ flow control and efficiency analysis for the HSDPA network. Legg [3] presented a strategy for the $I_{u b}$ link flow control, which found the scheduler in Node-B has a major influence on the Node-B buffer. Necker et. al. [4] investigated the impact of the $I_{u b}$ flow control on the HSDPA system performance, which indicated larger protocol delay and resource grant update period between Node-B and RNC could deteriorate the IP packet delay characteristic. Toskala et. al. [5] studied the $\mathrm{I}_{\mathrm{ub}}$ link efficiency on the HSDPA air interface. None of the previous works investigated the interdependencies between the RAN and the air interface. The paper is organized as follows. The HSDPA protocol

Optus.

The work is jointly supported by an ARC linkage grant and the SingTel 
architecture is briefly introduced in section II. Section III describes the proposed flow control algorithm, and its corresponding Markov chain model. The $\mathrm{I}_{\mathrm{ub}}$ based transport techniques and simulation models are introduced in the section IV. Comparative simulation results are presented in section V. Section VI presents the conclusion.

\section{HSDPA PROTOCOL ARCHITECTURE}

The HSDPA user plane protocol architecture is shown in Fig.1. Radio link control (RLC) layer handles the segmentation and retransmission of user and control data. The MAC-d (dedicated medium access control) layer is retained in the RNC to handle transport channel switching. MAC-hs (hs: high speed) layer is located in Node-B and responsible for scheduling, priority handling, H-ARQ and selection of an appropriate format and resources etc. The HS-DSCH (High Speed Dedicated Shared Channel) is the transport channel that carries the actual user data. The HS-DSCH FP (Frame Protocol) handles the data transport between a RNC and a Node-B. For a HSDPA connection data received from a core network is handled by RLC and MAC-d layers by creating MAC-d PDUs (Protocol Data Unit) and encapsulating into HS$\mathrm{DSCH}$ frames for transmission on the $\mathrm{I}_{\mathrm{ub}}$ link.

\section{I $\mathrm{I}_{\mathrm{UB}}$ FLOW CONTROL ALGORITHM}

The flow control is an important technique used in packet switched networks to reduce the possibilities of a buffer overflow and poor link utilization. In a recently published 3GPP document the E-UTRAN architecture specifies the transmission delay between UE (User Equipment) and RNC should be lower than $10 \mathrm{~ms}$ [6], [7]. The delay limit on the downlink is expected to be even shorter than the uplink. To ensure a lower connection delay between a RNC and an UE on the downlink it is necessary to employ a flow control algorithm. Fig. 2 shows the basic operation of the proposed flow control algorithm operating between a Node-B and a $\mathrm{RNC}$. In this model the RNC maintains a large buffer which is updated via the core network. The Node-B maintains a minimum buffer which will enable peak transmission rate for HSDPA users on the air interface. The flow control algorithm updates the Node-B buffer based on the HSDPA connection throughput and demand.

In this section we present an analytical model to analyze the performance of the HSDPA air interface using a Markov chain model. For each user we allocate a fixed space in the Node-B

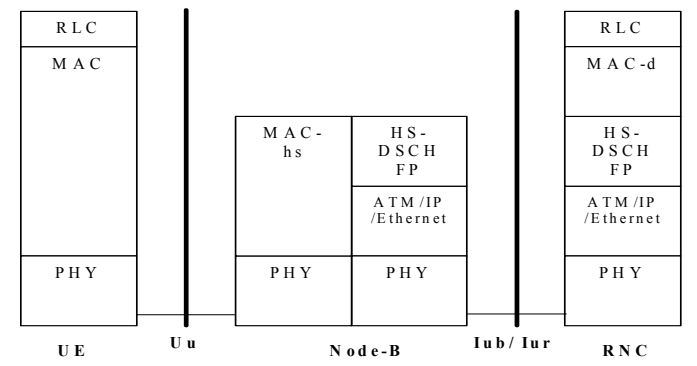

Figure 1. HSDPA protocol architecture

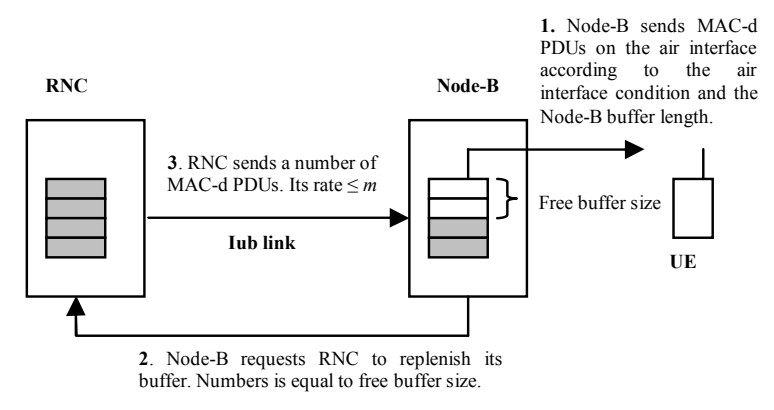

Figure 2. flow control betweeb Node-B and RNC

buffer. We assume that $n$ represents the buffer size in terms of MAC PDUs for each user. The value of $n$ is equal to total number of MAC-d PDUs that could be sent over the air interface at the peak rate in every TTI under the best transmission channel condition. We assume that the $I_{u b}$ link bandwidth is $m$, where $m$ is the actual number of MAC-d PDUs sent in each TTI over the link. After every transmission opportunities on the air interface, the Node-B immediately requests the RNC to send more MAC-d PDUs to replenish its buffer. The request size $R_{s}$ in number of PDUs is equal to the free buffer size. If the request size is larger than the $\mathrm{I}_{\mathrm{ub}}$ bandwidth $m$ i.e. $R_{s}>m$, then to avoid the $\mathrm{I}_{\mathrm{ub}}$ link congestion only $m$ MAC-PDUs are delivered to the Node-B. The value of $R_{s}$ could vary between 1 to $n$. To avoid a low $\mathrm{I}_{\mathrm{ub}}$ link utilization we maintain the following relationship $m<n$. Using the flow control algorithm the Node-B buffer is used to adjust the HSDPA user throughput on the air interface based on the radio link condition. It is possible that a peak air interface transmission rate may not be sustainable due to $\mathrm{I}_{\mathrm{ub}}$ link and the air interface transmission rate mismatch. Readers are reminded that the air interface throughput is radio channel condition dependent and varies over the time.

We present a brief analysis to calculate the HSDPA transmission channel efficiency. We define the HSDPA transmission channel efficiency $\eta$ as the ratio of actual number of MAC PDUs that can be transmitted via the air interface to the maximum number of MAC PDUs that can be supported by air interface condition per TTI, as shown in (1). The HSDPA transmission efficiency takes account of the radio channel condition, buffer length and the $\mathrm{I}_{\mathrm{ub}}$ link effective bandwidth. For example, on one occasion, if 4 PDUs of a HSDPA connection is in Node-B buffer but the radio link condition permits transmission of 10 PDUs then we obtain a $\eta$ value of $0.4(4 / 10)$. In this case when a transmission opportunity arises then a Node-B can not transmit sufficient number PDUs. This situation can be avoided by increasing the fixed allocation of bandwidth on the $I_{u b}$ link but that will reduce the $I_{u b}$ link efficiency.

$$
\eta=\frac{A C T U A L_{-} M A C_{-} P D U_{-} T x}{M A X_{-} M A C_{-} P D U_{-} \text {air }}
$$

We assume that the transmission rate (expressed in number of MAC-d PDUs of per TTI) of a HSDPA connection is distributed between 0 and to a maximum number $n$. The value of $n$ depends on the UE category. Let $r$ represent the air 
interface rate, which a radio channel condition can support. Let $R_{i}$ represents the probability of air interface transmission rate, where $i$ represent the number of PDUs transmitted at that rate. After every transmission opportunity the Node-B buffer is replenished. Let $l_{n}$ be MAC-d PDU numbers in Node-B buffer measured after the buffer is replenished and waiting for the next transmission opportunity in $n$-th TTI. The buffer length could vary between $m$ and $n$, i.e. $m \leq l_{n} \leq n$. We can form a Markov chain using the values of $l_{n}$. Consider the stationary state where all $\left\{l_{n}\right\}$ have the same probability distribution. Let $l$ denote the random variable in MAC-d PDU numbers in the Node-B buffer.

$$
\text { Let, } \pi_{i}=P\{l=m+i\}, \quad i=0, \ldots ., n-m
$$

The transition matrix is shown in (3).

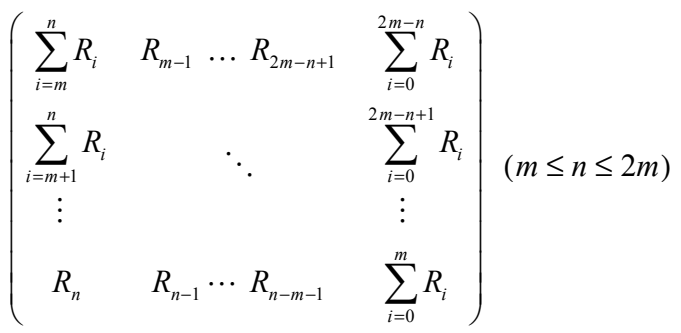

In the stationary state, we can write $\pi=\pi P$, so

$$
\begin{aligned}
& \pi_{0}=\pi_{0} \sum_{i=m}^{n} R_{i}+\pi_{1} \sum_{i=m+1}^{n} R_{i}+\ldots .+\pi_{n-m} R_{n} \\
& \pi_{1}=\pi_{0} R_{m-1}+\pi_{1} R_{m}+\ldots .+\pi_{n-m} R_{n-1} \\
& \vdots \\
& \pi_{n-m-1}=\pi_{0} R_{2 m-n+1}+\pi_{1} R_{2 m-n}+\ldots .+\pi_{n-m} R_{n-m-1} \\
& \pi_{n-m}=\pi_{0} \sum_{i=0}^{2 m-n} R_{i}+\pi_{1} \sum_{i=0}^{2 m-n+1} R_{i}+\ldots .+\pi_{n-m} \sum_{i=0}^{m} R_{i} \\
& \pi_{0}+\pi_{1}+\cdots+\pi_{n-m}=1
\end{aligned}
$$

Recalling the HSDPA link efficiency definition as shown in (1), we calculate the HSDPA transmission efficiency. Equation (5) shows the generalized form the efficiency equation.

$$
\begin{aligned}
& \text { When } l=m, \\
& \text { if } r \leq m, \eta_{l=m}=100 \% \text {; } \\
& \text { if } r=m+1, \eta_{l=m}=\frac{m}{m+1} \text {; } \\
& \vdots \\
& \text { if } r=n, \eta_{l=m}=\frac{m}{n} ; \\
& \vdots \\
& \text { When } l=n, \\
& \eta_{l=n}=100 \%
\end{aligned}
$$
by:

So, the average HSDPA transmission efficiency is given

$$
\begin{aligned}
\eta= & \pi_{0}\left(\sum_{i=0}^{m} R_{i} \times 1+R_{m+1} \times \frac{m}{m+1}+\ldots .+R_{n} \times \frac{m}{n}\right)+ \\
& \pi_{1}\left(\sum_{i=0}^{m+1} R_{i} \times 1+R_{m+2} \times \frac{m+1}{m+2}+\ldots .+R_{n} \times \frac{m+1}{n}\right) \\
& +\ldots .+\pi_{n-m} \times 1
\end{aligned}
$$

From the statistical point of view, the air interface capacity of a cell will be approximately normally distributed according to the Central Limit Theorem. Since the distribution of air interface rate is discrete, to simplify our analysis, we approximate the HSDPA air interface data rate using the following binomial distribution (6).

$$
\begin{aligned}
& R_{i}=\left(\frac{n}{i}\right) p^{i}(1-p)^{n-i} \quad 0<i \leq n ; 0<p \leq 1 \\
& \text { mean }=n \cdot p \\
& \text { HSDPA average throughput at RLC layer } \\
& =n \times p \times R L C \text { _Packet_size/TTI }
\end{aligned}
$$

Where $R_{i}$ represents the probability of air interface transmission rate, $i$ represent the number of PDUs transmitted at that rate and $n$ is equal to the maximum number of MAC-d PDUs that can be sent in a TTI. The value of $p$ depends on cell parameters. We assume that a very good and controlled coverage in a small cell with low user mobility could lead to higher value of $p$ representing higher air interface transmission rate for users. Best radio channel condition is represented by the value of $p=1$. Fig. 3 shows the probability distribution of air interface transmission rate for $p=0.8$, and $n=10$ for a category 12 UE. This plot shows that for $p=0.8$ a HSDPA link may transmit a number of PDUs per TTI at different times. The plot shows that there are higher probabilities that a UE will transmit 7 to 9 PDUs per TTI. The peak of the plot will shift towards left for lower values of $p$. Similar results were reported by an Ericsson research group who measured HSDPA network performances in different transmission conditions [8].

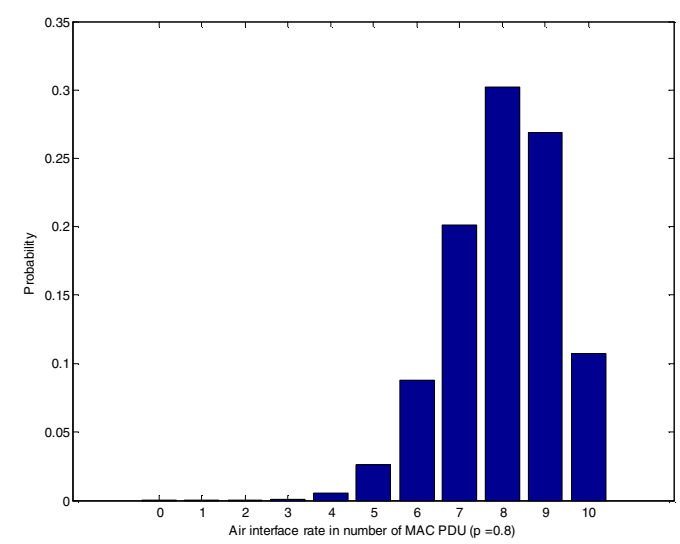

Figure 3. Probability distribution of air interface represented in number of MAC-d PDUs for $p=0.8$ and $\mathrm{n}=10$ 
Using the above analytical technique, we can calculate the HSDPA transmission efficiency for different cell parameters. For a category $12 \mathrm{UE}$, the air interface transmission rate is distributed between 0 to 10 MAC-d PDUs. These PDUs are transmitted from the Node-B according to the current air interface data rate. Since $\mathrm{I}_{\mathrm{ub}}$ link effective bandwidth $m$ is 8 MAC-d PDU per TTI, at least 8 PDUs are waiting in Node-B buffer for transmission in the next turn. The size of Node-B buffer $l$ could vary between $m$ to $n$. Using above values a transition matrix can be constructed. The transition matrix $P$ is shown by (9). Table I shows the HSDPA theoretical transmission efficiency for different values of $p, l$ and air interface average throughput. These values are calculated for a single cell with a fix RLC/MAC PDU size of 320 bits with a maximum air interface capacity of 10 PDUs per TTI. An AAL2/ATM $\mathrm{I}_{\mathrm{ub}}$ link of $2.048 \mathrm{Mbs}$ is used. The value of $m$ is set to 8 .

$$
P=\left(\begin{array}{ccc}
\sum_{i=8}^{10} R_{i} & R_{7} & \sum_{i=0}^{6} R_{i} \\
\sum_{i=9}^{10} R_{i} & R_{8} & \sum_{i=0}^{7} R_{i} \\
R_{10} & R_{9} & \sum_{i=0}^{8} R_{i}
\end{array}\right)
$$

These results show that for an ATM based $\mathrm{I}_{\mathrm{ub}}$ link higher HSDPA efficiency $\eta$ can be achieved for lower values of $p$. The value of $\eta$ decreases with the increasing value of $p$ because for a better channel condition the average value of $R_{s}$ (the request size) becomes larger than $m$, hence, the $\mathrm{I}_{\mathrm{ub}}$ link is unable to supply sufficient number of MAC-PDUs. The effect of the $I_{u b}$ link characteristics is also influenced by the scheduling mechanism at Node-B. For example, a round robin (RR) scheduler will impose less restriction on the $\mathrm{I}_{\mathrm{ub}}$ link than $\mathrm{a}$ proportional fair (PF) scheduling scheme. Because using a PF scheduler the same UE can receive multiple transmission opportunities in successive TTIs.

\section{Simulation Model}

An OPNET based simulation model has been developed to obtain simulation results. The following section briefly describes the simulator structure.

TABLE I. CALCUlation Results (UE CAT.12)

\begin{tabular}{|c|c|c|c|c|c|c|c|}
\hline \multicolumn{2}{|c|}{$p$} & 0.5 & 0.6 & 0.7 & 0.8 & 0.9 & 1.0 \\
\hline \multicolumn{2}{|c|}{$\begin{array}{c}\text { Air interface } \\
\text { average } \\
\text { throughput } \\
\text { (kbps) }\end{array}$} & 800 & 960 & 1120 & 1280 & 1440 & 1600 \\
\hline \multirow{3}{*}{$\pi$} & $l=8$ & 0.001 & 0.009 & 0.072 & 0.406 & 0.902 & 1 \\
\hline & $l=9$ & 0.010 & 0.046 & 0.148 & 0.249 & 0.075 & 0 \\
\hline & $l=10$ & 0.989 & 0.945 & 0.780 & 0.345 & 0.023 & 0 \\
\hline \multicolumn{2}{|c|}{$\eta(\%)$} & $100 \%$ & 99.99 & 99.82 & 97.65 & 89.57 & 80 \\
\hline
\end{tabular}

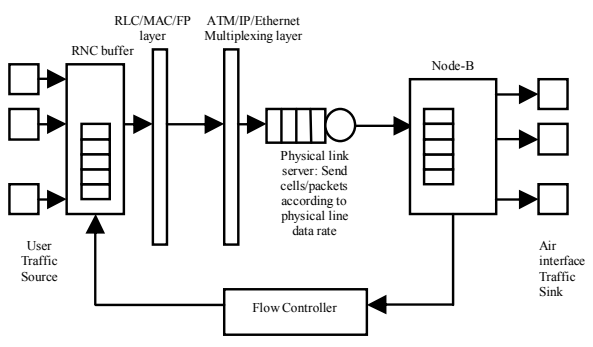

Figure 4. The OPNET simlator structure

\section{A. Simulator Architecture}

The simulator is consists of an $\mathrm{I}_{\mathrm{ub}}$ interface and a physical link, a traffic generator, an air interface link and the proposed flow controller as shown in the Fig. 4. The flow controller is implemented according to the description of the section III. The Node-B allocates each HSDPA connection a transmission bandwidth according to the radio channel condition which is simulated by the binomial distribution as shown in the Fig. 3. In the OPNET model the air interface actions were simulated by traffic sinks as shown on the right side of the figure. At the Node-B RLC/MAC PDUs are extracted and stored in the Node-B buffer for transmission on the downlink [9], [10]. In this model three different $\mathrm{I}_{\mathrm{ub}}$ transport protocols were used. Those are AAL2/ATM, CIP (Composite IP) [11] and the carrier class Ethernet [12].

\section{B. Traffic Model}

In this work we initially concentrated on a bursty web traffic transmission. The UMTS traffic model is used to develop the web browsing application [11]. The generated web page file size is Pareto distributed with a parameter $\alpha=1.1$, mean file size of 12,000 bytes, minimal file size of $k=1858$ bytes and the maximum file size $m=5000000$ bytes. The probability density function of the Pareto distribution is shown in (10).

$$
f(x)=\left\{\begin{array}{lr}
\frac{\alpha \cdot k^{\alpha}}{x^{\alpha+1}}, & k \leq x \leq m \\
\frac{k^{\alpha}}{m^{\alpha}}, & x>m
\end{array}\right.
$$

\section{Simulation Results}

A number of simulation results are presented in this section. Fig. 5 shows the relationship between the value of $p$ and $\eta$ for category 12 UE. The plot shows that theoretical and simulation results are in very close agreement. The figure shows that the value of $\eta$ decreases as the transmission channel quality improves because the $I_{u b}$ link creates the bottleneck. Next we observe the air interface and $\mathrm{I}_{\mathrm{ub}}$ link throughputs for different channel conditions. Fig. 6 shows that the HSDPA air interface throughput can linearly increase with the increasing value of $p$. However, due to the $\mathrm{I}_{\mathrm{ub}}$ link bandwidth constraint, the air interface throughput remains limited at $1.3 \mathrm{Mbps}$ for a 2.048 Mbps ATM based $I_{u b}$ link. On the other hand the graph also shows that the $\mathrm{I}_{\mathrm{ub}}$ link utilization remains low for lower values of $p$ because of lack of demand by the air interface. In this case 
if a higher bandwidth is used for the $\mathrm{I}_{\mathrm{ub}}$ then the link utilization will fall. So, these results show that simply by increasing the $I_{u b}$ link capacity it is not possible to improve the overall system efficiency. Next we examine the Node-B buffer occupancy levels for different air interface transmission conditions as shown in Fig. 7. The figure shows both analytical and simulation results. The figure clearly shows that they are in very close agreement. The graph shows that for $p=0.7$ the buffer occupancy level is 10 MAC PDU for about $80 \%$ of the time whereas for $p=0.9$ for about $90 \%$ time the buffer length is 8 the minimum buffer size. The reason for shorter buffer length for $p=0.9$ is that the Node-B drain rate by the air interface is higher than the buffer replenishment rate. The buffer length is measured after every transmission on the air interface and its subsequent buffer replenishment by the RNC. The graph shows that $p=0.8$ is a balanced region where drain rate is matched by the replenishment rate. Finally, we compare three $I_{u b}$ link transport protocols as shown in Fig. 8. Result shows that the CIP solution offers the best HSDPA efficiency figures for both average and good channel conditions. Results also show that the increased $\mathrm{I}_{\mathrm{ub}}$ link data rate improves the value of $\eta$ for different transmission channel conditions.

\section{CONCLUSIONS}

In this paper we presented novel analytical and simulation techniques to study the relationships between the HSDPA air interface and the $\mathrm{I}_{\mathrm{ub}}$ link characteristics. For next generation HSDPA services it is necessary to properly dimension radio access networks to support high data rate multimedia services on an air interface. The results can be used as a reference for network designers.

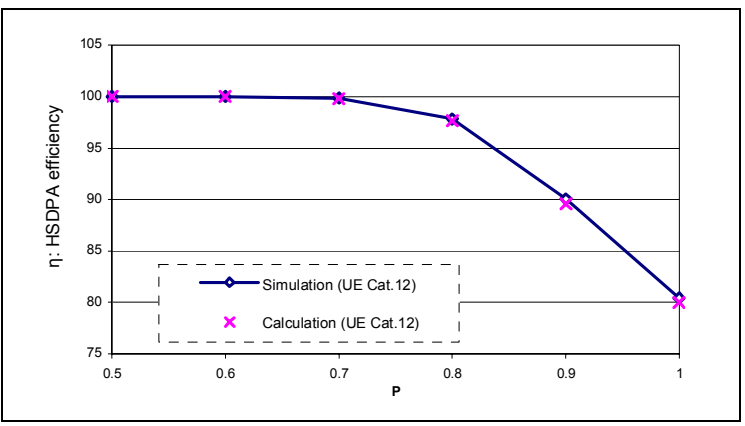

Figure 5. HSDPA efficiency for different values of $p$

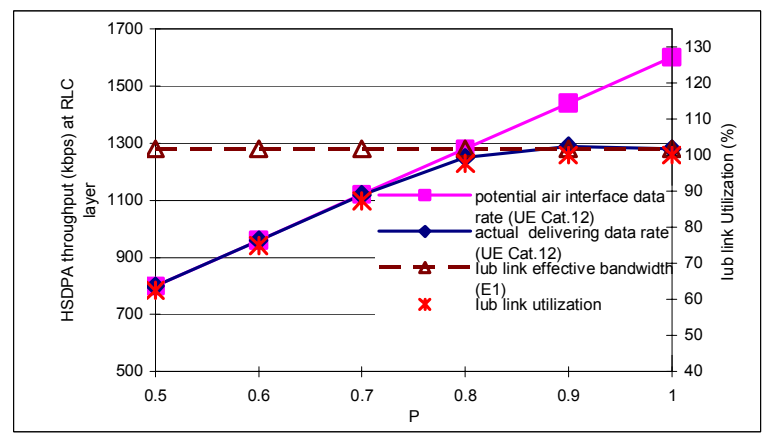

Figure 6. HSDPA average throughput (RLC layer) and $\mathrm{I}_{\mathrm{ub}}$ link utilization

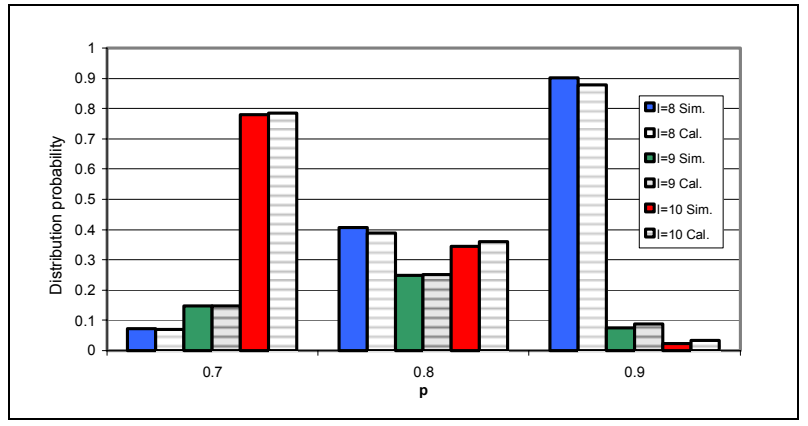

Figure 7. Distribution number of PDUs in the Node-B buffer for UE Cat.12

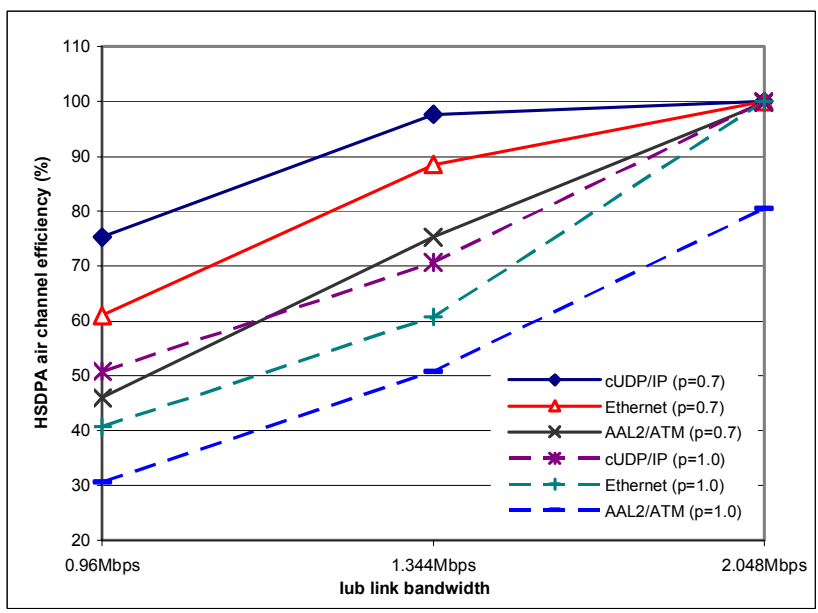

Figure 8. HSDPA air interface efficient with $\mathrm{I}_{\mathrm{ub}}$ physical link bandwidth

\section{REFERENCES}

[1] E. Dahlman, et.al, "The 3G Long-term Evolution- Radio Interface Concepts and Performance Evolution", 2006 IEEE 63rd Vehicular Technology Conference, Melbourne, Australia, 7-10 May, 2006.

[2] H. Holma, A. Toskala, HSDPA/HSUPA for UMTS, John Wiley and Sons, 2006, pp.26-27.

[3] P.J.Legg, "Optimised Iub flow control for UMTS HSDPA" Proc. of the IEEE VTC 2005-Spring, Stockholm, Sweenden, Jun 2005.

[4] M. C. Necker and A. Weber, "Impact of lub flow control on HSDPA system performance," Proc. PIMRC 2005, Berlin, Germany, September 2005 .

[5] A. Toskala, H.Holma, E. Metsala, K. I. Pedersen, and D. Steele, "Iub efficiency analysis for high speed downlink packet access in WCDMA", Proc. WPMC, Aalborg, Denmark, September, 2005.

[6] 3GPP 25.913 "Requirements for Evolved UTRA(E-UTRA) \& Evolved UTRAN (E-UTRAN)”, Relese 7, v.7.3, 03/2006.

[7] A. Toskala, et. al. "UTRAN Long Term evoltion in 3GPP", Proc. PIMRC 2006, helsinki, Finland, September 2006.

[8] J.Derksen, R. Jansen, M. Maijala, and E. Westerberg. "HSDPA performance and evolution", Ericsson Review, Vol.3, 2006.

[9] 3GPP TS 25.308 v6.3.0 (2004-12), "High Speed Downlink Packet Access (HSDPA); Overall description; Stage 2 (Release 6)".

[10] 3GPP TS 25.435 v6.3.0, "UTRAN Iub Interface User Plane Protocols for Common Transport Channel data streams (Release 6)"

[11] 3GPP TR 25.933 “IP transport in UTRAN (Release 5)".

[12] ITU-T G.8040/Y.1340, "GFP frame mapping into Plesiochronous Digital Hierarchy (PDH)”. 